\title{
Testing the ability for autonomous oral hygiene in hospitalized geriatric patients-clinical validation study
}

\author{
Ina Manuela Schüler ${ }^{1}$ (D) $\cdot$ Barbara Kurtz ${ }^{1} \cdot$ Roswitha Heinrich-Weltzien $^{1} \cdot$ Thomas Lehmann $^{2} \cdot$ Anja Kwetkat $^{3}$
}

Received: 24 April 2019 / Accepted: 10 June 2020 / Published online: 23 June 2020

(C) The Author(s) 2020

\begin{abstract}
Objectives This study aimed to evaluate if the Timed Test for Money Counting (TTMC) complemented with testing the range of shoulder motion by griping the backside of the neck (NG) predicts the ability of geriatric inpatients to perform effective plaque reduction by autonomously conducted oral hygiene.

Material and methods This clinical validation study involved 74 hospitalized geriatric inpatients, 48 (64.9\%) females, aged between 66 and 98 years (mean age 84.1 years). Oral health status was examined. Dental plaque was assessed with the Turesky modified Quigley-Hein Index (TI) on teeth and the Denture Hygiene Index (DHI) on removable dentures. The performance and duration of TTMC and NG were recorded. After autonomous tooth brushing and denture cleaning by the patient, dental plaque was scored again with the TI and DHI. Geriatric assessment data were collected from medical records.

Results Forty-nine (66.2\%) geriatric inpatients completed the TTMC\&NG successfully. Passing the TTMC\&NG was significantly associated with better plaque removal on teeth and dentures by autonomously conducted oral hygiene. The sensitivity of the TTMC\&NG for above average plaque reduction was $86.4 \%$ on teeth and $77.8 \%$ on dentures. The test revealed a negative predictive value of $75.0 \%$ to detect below average plaque reduction on teeth and $72.7 \%$ on dentures.

Conclusions The TTMC\&NG served as a suitable predictor for the ability of geriatric inpatients to perform autonomously effective tooth brushing and denture cleaning.

Clinical relevance This simple and short test might help the medical staff to identify geriatric inpatients unable to perform effective oral hygiene by themselves.
\end{abstract}

Keywords Oral hygiene $\cdot$ Geriatric assessment $\cdot$ Self-management $\cdot$ Timed Test for Money Counting

\section{Introduction}

Hospitalized geriatric patients bear a double burden — besides the functional and cognitive limitations accompanying the aging process they have to cope with multimorbidity and acute medical conditions. In this situation, efforts should be

Ina Manuela Schüler

Ina.Schueler@med.uni-jena.de

1 Section of Preventive and Pediatric Dentistry, Department of Orthodontics, Jena University Hospital, Bachstraße 18, D -, 07743 Jena, Germany

2 Institute of Medical Statistics, Computer Sciences and Documentation, Jena University Hospital, Jena, Germany

3 Department of Geriatric Medicine, Jena University Hospital, Jena, Germany undertaken to avoid additional concomitant oral healthrelated problems due to insufficient oral hygiene.

Maintaining good oral health is important for geriatric patients because it is strongly related to nutrition intake, decreased risk of respiratory and cardiovascular diseases, and better quality of life [1-4]. Furthermore, chronic periodontal inflammations persisting over many years are assumed to promote the inflammaging process, increasing the susceptibility for infections, malignancies, and autoimmune diseases [5]. Nevertheless, studies report high prevalence rates of dental caries, periodontal disease, tooth loss, and mucositis [6-8]. Gerontologists are concerned that poor oral health will become a new geriatric syndrome [9].

Oral cleanliness is crucial for maintaining oral health. Neglect of oral hygiene threatens the patients' well-being, and general health might become jeopardized [10]. Plaqueinduced oral diseases were identified as a major risk to a patient's ability to eat, communicate, and socialize [11]. 
Furthermore, dental plaque might influence the course of respiratory infections $[2,12,13]$. There is good evidence that effective plaque removal reduces progression or occurrence of respiratory diseases among frail elderly nursing home residents, hospitalized patients, and especially intensive care patients $[2,14-16]$.

Regarding frail and medically compromised geriatric patients, the question arises which patients are still capable to perform sufficient oral hygiene by themselves and which patients need assistance or oral hygiene to be overtaken by nursing staff. This decision is reported to be difficult for dentists [17] and probably even more challenging for geriatric medical and nursing staff. A short and easy-to-use test for detecting geriatric patients with impaired ability to perform effective oral hygiene by non-dental professionals might help in this decision process.

Tooth brushing and denture cleaning require a certain level of manual dexterity, visual acuity, procedural and cognitive skills, and sufficient mobility of the shoulder and elbow joints. The Timed Test for Money Counting (TTMC) is a validated and established geriatric assessment tool evaluating manual skills, visual acuity, and cognitive capacity [18]. It is simple to perform and requires about $5 \mathrm{~min}$. The TTMC was successfully validated to evaluate geriatric patients' ability to manage insulin therapy autonomously [19], which requires similar skills as oral hygiene. Except the shoulder mobility, all general skills necessary to perform oral hygiene might be assessed with the TTMC. The range of shoulder mobility can be tested short and easily inviting the patient to grip the backside of the neck (NG). The combination of both tests-TTMC\&NGcovers the main skills necessary to perform tooth brushing and denture cleaning.

The aim of this study was to evaluate if the TTMC\&NG could serve as a suitable tool for testing the ability of geriatric inpatients to perform effective plaque reduction by autonomous tooth brushing and denture cleaning.

\section{Material and methods}

This clinical validation study was performed between May 2016 and May 2017 at the Geriatric Clinic of Jena University Hospital.

\section{Study sample}

All patients hospitalized during the study period were eligible to enrollment. The mean age was used to divide the study sample in one younger (under mean) and one older (over mean) age group.

The sample size was estimated to the number of patients necessary to evidence a strong association between passing or failing the TTMC\&NG and plaque reduction rates on teeth and dentures with an effect size of $d \geq 0.5,90 \%$ power, and alpha $=0.05$. Forty-two patients with natural teeth and 42 with removable dentures had to be included.

\section{Oral examination}

All patients were examined in the patient rooms of the geriatric ward by one calibrated dentist (B.K.) using a ball-end probe and an illuminated mirror (DenLite ${ }^{\circledR}$, Miltex, USA) after drying the teeth with cotton rolls and without use of radiographs. For examination, patients were sitting on a chair or lying in bed in supine position.

Dental caries was scored in accordance with the WHO criteria for caries diagnostics [20] using the DMFT index.

Gingival health was evaluated by the periodontal screening index (PSI) [21]. The gingiva surrounding all teeth was examined with a standardized ball-end probe with a black band from 3.5 to $5.5 \mathrm{~mm}$ using light probing force. After dividing the dentition into sextants, the highest score of each sextant was recorded.

Dental plaque was scored in all patients with natural tooth crowns by Turesky modified Quigley-Hein Index (TI) [22] after staining all teeth surfaces with Rondells Blue $\odot$ (Directa, Sweden) before ( $\mathrm{t} 0$ ) and after autonomously tooth brushing (t1). Tooth brushing was performed in the washing facilities of the patients' rooms. Plaque on dentures was scored in all patients with removable dentures by Denture Hygiene Index (DHI) [23] by visual inspection without staining before ( $\mathrm{t} 0$ ) and after ( $\mathrm{t} 1)$ denture cleaning performed by the inpatient.

Prior to the examinations, the dentist (B.K.) was trained by an experienced dentist and epidemiologist (I.M.S.). The intraund inter-examiner reproducibility was assessed by the weighted kappa $\left(k_{\mathrm{w}}\right)$ statistics. The $k_{\mathrm{w}}$ values for interexaminer reproducibility ranged from 0.89 for the TI Index to 0.91 for the DHI. Intra-examiner reproducibility ranged from 0.94 to 0.97 (examiner B.K.) and 0.93 to 0.97 (examiner I.M.S.) for both indices. The inter- and intra-examiner reproducibility for the TTMC\&NG was 1.0 for both examiners.

\section{Timed Test for Money Counting}

The Timed Test for Money Counting (TTMC) measures the time a person needs to open a standardized purse, to take out the standardized amount of money in a standardized denomination, and to count it with the aim to identify persons in need for increased health care [18].

In the present study, a conventional purse (about $12 \times 9 \mathrm{~cm}$ ) with one compartment for bills and one with push button for coins contained $9.80 €$ in the following denominations: $1 \times 5 €$ note in the compartment for bills and $1 \times 2 €$ coin, $2 \times 1 €$ coins, $1 \times 50$ cent coin, and $3 \times 10$ cent coins in the compartment for coins. 
The purse was shown to the patient; it was closed again and handed over to the patient, with the request to count the money in the purse. The time was measured in seconds with a stopwatch. If the result was correct, the time was stopped and noted. If an incorrect result was reported, the patient was notified that the finding was incorrect and she/he was allowed to try again. The time measurement continued. The test was scored as failed after three failed attempts, when more than $300 \mathrm{~s}(5 \mathrm{~min})$ were needed or if the patient canceled the test due to lack of self-perceived ability or visual or motoric problems. The TTMC uses cutoff times of less than $45 \mathrm{~s}$ to identify independent patients, $45-70 \mathrm{~s}$ for patients at risk for neediness, and more than $70 \mathrm{~s}$ for patients at risk for extensive assistance $[18,24]$. Additionally, difficulties related to fine and gross motor skills and visual and cognitive limitations during test performance were recorded.

\section{Range of movement for shoulder motion}

Restricted range of shoulder motion was detected by asking the patients to grip the backside of their neck (NG) while standing or sitting in an upright position without props. The examiner decided if the shoulder range of movement was sufficient to reach the backside of the neck or not. It was precluded that patients who are able to reach their own backside of the neck with both hands or at least with the dominant hand are also able to reach their oral cavities for tooth brushing. Complaint of pain was recorded.

The combination of TTMC\&NG was scored as failed, if one of the tests were not completed successfully.

\section{Oral health self-assessment questionnaire}

A simple paper-based questionnaire with mainly closed questions was used to record the patient's self-perceived oral health, oral hygiene habits, and independency of oral hygiene at home and at the hospital. Furthermore, patients were asked if they perceive having problems in performing oral hygiene by themselves. Wording of the questions was concise, unambiguous, and asked in a personal interview.

\section{Collection of geriatric assessment data}

Comprehensive geriatric assessment is part of the daily routine by geriatric physicians or medical staff and conducted with validated and standardized tools. It is a multidimensional interdisciplinary diagnostic process focused on determining patients' medical, psychological, and functional capability aiming to develop a coordinated and integrated plan for treatment and long-term follow-ups [25]. In this study, the results of standard geriatric assessment tests (Barthel Index [26], Geriatric Depression Scale by Yesavage [27], and Mini Mental State Examination by Folstein [28]) conducted in daily routine by geriatric staff were collected from the medical records of the patients.

The Barthel Index has been developed to assess basic everyday functions in patients with neurological or musculoskeletal impairment [26]. The objective is to determine the degree of independence concerning the two main areas of self-sufficiency: personal care (food intake, personal hygiene, toilet use) and mobility (locomotion, climbing stairs). Higher score indicates greater independence of the patient but only for the activities observed in the test. A score of 100 points characterized an independent patient, 95-85 points a patient requiring low-level care, 80-35 points a patient needing medium-level care, and 30-0 points a patient demanding high-level care [29].

Cognitive function was assessed using the German version of the Mini Mental State Examination (MMSE) [28], testing cognitive aspects of mental function, e.g., orientation, registration, attention, memory, and ability to follow verbal and written commands. The MMSE has a score ranging between 0 and 30 . The cognitive impairment is assumed severe with a score $\leq 17$ points and as mild with a score of 18-23 points. Patients achieving scores of 24-30 points were considered without significant cognitive impairment [30].

The German version of the Geriatric Depression Scale after Yesavage (GDS) [27] was used to detect depressed mood and signs of depressive disorders. It consists of 15 questions, and each answer indicating depressive mood is counted. The score ranges from 0 to 15 . A score of $0-4$ is considered to be within the normal range, 5-9 indicates mild depression, and score 10 or more indicates moderate to severe depression [31].

\section{Data analysis and statistics}

Data collection was performed with Microsoft Excel® 2013 (Microsoft Corp., Redmond, USA) and statistical analysis by SPSS for Windows (IBM SPSS Statistics for Windows, Version 20.0. Armonk, NY: IBM Corp). Mean values were analyzed by $t$ test and ANOVA and categorical data by chisquare test. Spearman's rho was calculated to detect correlation between continuous variables.

For test validation, $2 \times 2$ tables were used to assign true positive (TP), true negative (TN), false positive (FP), and false negative (FN) test results and to calculate sensitivity (SE), specificity (SP), positive predictive value (PPV), and negative predictive value (NPV) by following formulas: $\mathrm{SE}=\mathrm{TP} /$ $(\mathrm{TP}+\mathrm{FN}) \times 100, \mathrm{SP}=\mathrm{TN} /(\mathrm{TN}+\mathrm{FP}) \times 100), \mathrm{PPV}=\mathrm{TP} /$ $(\mathrm{TP}+\mathrm{FP}) \times 100$, and $\mathrm{NPV}=\mathrm{TN} /(\mathrm{FN}+\mathrm{TN}) \times 100$.

Significance level was set at $p \leq 0.05$. 


\section{Results}

From 809 eligible patients, $74(9.2 \%)$ hospitalized geriatric inpatients aged between 66 and 98 years with mean age of $84.1(\mathrm{SD}=5.8)$ years were included, $64.9 \%$ were females. Causes for nonparticipation were severe medical conditions, quarantine due to infections or colonization with multiresistant pathogens, or lack of willingness. In the study population, 46 patients had natural teeth; two of them had only tooth roots without crowns impeding the assessment of TI. Thirty-seven of the dentate patients and all 28 toothless patients wore removable dentures. Due to the clinically frequently met situation of dentate patients wearing partial dentures, the number of patients with removable dentures $(n=63)$ exceeded the required sample size.

\section{Oral health}

Acute dental treatment need was diagnosed in $60.8 \%$ of the patients, while acute oral pain was reported by $9.5 \%$ (Table 1).
All results concerning oral health are shown in Table 1. Within the study population, $62.2 \%$ were dentate with a mean number of 6.9 remaining teeth. More patients aged over 84 years were edentulous compared with those up to 84 years. The number of teeth was higher in patients up to 84 years and in females. Caries experience revealed no significant age- or sex-related differences but in means 1.7 teeth with untreated carious lesions.

Prevalence of dentures was $85.1 \%$. Complete dentures were more frequently in the upper $(54.1 \%)$ than in the lower jaw $(41.9 \%)$.

Gingivitis and periodontitis increased by age and gingivitis occurred more often in males and periodontitis in females, both without statistical significance. Slightly more dental plaque was recorded in older patients compared with the younger age group and in males compared with females.

The majority of patients evaluated their oral hygiene as good, females slightly more frequently than males. Problems in performing oral hygiene were reported by $16.2 \%$ of the patients, more often by males compared with females. The majority of patients performed oral hygiene at home or in the hospital without any help.

Table 1 Oral health parameters in geriatric patients

\begin{tabular}{|c|c|c|c|c|c|}
\hline & All & Age $\leq 84$ years & Age $>84$ years & Males & Females \\
\hline Study population \% (n) [95\% CI] & $100.0(74)$ & $52.7(39)[41.9-63.5]$ & $47.3(35)[36.5-58.1]$ & $35.1(26)[24.3-45.9]$ & $64.9(48)[54.1-75.7]$ \\
\hline \multicolumn{6}{|c|}{ Dental status } \\
\hline Dentate $\%(n)[95 \% \mathrm{CI}]$ & $62.2(46)[50.0-73.0]$ & $71.8(28)[56.8-85.0]$ & $51.4(18)[34.5-69.2]$ & $65.4(17)[47.4-83.3]$ & $60.4(29)[46.3-73.3]$ \\
\hline Number of teeth MW (SD) & $6.9(0.0)$ & $8.4(8.8)$ & $5.3(6.8)$ & $6.4(7.2)$ & $7.3(8.5)$ \\
\hline DMFT MW (SD) & $26.3(3.1)$ & $26.2(3.4)$ & $26.5(2.7)$ & $26.4(2.4)$ & $26.3(3.4)$ \\
\hline Dentures \% $(n)[95 \% \mathrm{CI}]$ & $85.1(63)[75.7-93.2]$ & $79.5(31)[66.7-91.4]$ & $91.4(32)[81.8-100.0]$ & $84.6(22)[70.8-96.0]$ & $85.4(41)$ [74.1-95.0] \\
\hline Acute oral pain $\%(n)[95 \% \mathrm{CI}]$ & $9.5(7)[2.7-16.2]$ & $10.3(4)[2.2-20.0]$ & $8.6(3)[0.0-18.9]$ & $15.4(4)[3.6-32.0]$ & $6.3(3)[0.0-14.3]$ \\
\hline $\begin{array}{l}\text { Acute dental treatment need } \\
\%(n)[95 \% \mathrm{CI}]\end{array}$ & $60.8(45)[50.0-71.6]$ & $69.2(27)[55.3-83.3]$ & $51.4(18)[34.4-67.7]$ & $57.7(15)[38.1-76.7]$ & $62.5(30)[48.0-75.5]$ \\
\hline \multicolumn{6}{|l|}{ Periodontal status } \\
\hline $\mathrm{PSI}=0 \%(n)[95 \% \mathrm{CI}]$ & $10.9(5)[2.2-21.7]$ & $17.9(5)[4.2-33.3]$ & $0.0(0)[0.0-0.0]$ & 5.9 (1) $[0.0-18.8]$ & 13.8 (4) [3.1-26.7] \\
\hline $\mathrm{PSI}=1-2 \%(n)[95 \% \mathrm{CI}]$ & 45.7 (21) [32.6-58.7] & 39.3 (11) [21.2-58.6] & $55.6(10)[31.6-81.2]$ & 58.8 (10) [33.3-84.2] & 37.9 (11) [20.8-57.7] \\
\hline $\mathrm{PSI}=3-4 \%(n)[95 \% \mathrm{CI}]$ & $43.5(20)[28.3-58.7]$ & 42.9 (12) [23.1-62.1] & $44.4(8)$ [18.8-68.4] & $35.3(6)[13.4-60.0]$ & 48.3 (14) [30.8-66.7] \\
\hline \multicolumn{6}{|l|}{ Dental plaque } \\
\hline TI at t0 MW (SD) & $2.74(1.07)$ & $2.61(1.12)$ & $2.94(1.00)$ & $3.11(1.17)$ & $2.53(0.96)$ \\
\hline DHI at t0 MW (SD) & $0.45(0.31)$ & $0.44(0.32)$ & $0.47(0.3)$ & $0.53(0.36)$ & $0.41(0.28)$ \\
\hline \multicolumn{6}{|l|}{ Self-evaluation } \\
\hline $\begin{array}{l}\text { My oral hygiene is very } \\
\text { good } \%(n)[95 \% \mathrm{CI}]\end{array}$ & $5.4(4)[1.4-10.8]$ & $5.1(2)[0.0-13.0]$ & 5.7 (2) $[0.0-14.3]$ & 7.7 (2) $[0.0-19.0]$ & $4.2(2)[0.0-10.6]$ \\
\hline $\begin{array}{l}\text { My oral hygiene is } \\
\text { good } \%(n)[95 \% \mathrm{CI}]\end{array}$ & $68.9(51)[58.1-78.4]$ & $69.2(27)[53.1-83.3]$ & $68.6(24)[53.1-84.2]$ & 61.5 (16) [40.6-80.6] & $72.9(35)[60.0-84.8]$ \\
\hline $\begin{array}{l}\text { My oral hygiene is satisfactory } \\
\%(n)[95 \% \mathrm{CI}]\end{array}$ & 25.7 (19) [16.2-36.5] & $25.6(10)[13.3-41.2]$ & 25.7 (9) [12.1-40.6] & $30.8(8)$ [13.3-50.0] & $22.9(11)[11.8-35.6]$ \\
\hline $\begin{array}{l}\text { I have problems in performing } \\
\text { oral hygiene } \%(n)[95 \% \mathrm{CI}]\end{array}$ & 16.2 (12) [8.1-25.7] & 17.9 (7) [7.0-31.6] & $14.3(5)[4.2-27.6]$ & 26.9 (7) [10.3-43.8] & $10.4(5)[2.3-20.0]$ \\
\hline $\begin{array}{l}\text { I perform oral hygiene without } \\
\text { help \% }(n)[95 \% \mathrm{CI}]\end{array}$ & 87.8 (65) [79.7-94.6] & $82.1(32)$ [6.5-31.2] & 94.3 (33) [84.8-100.0] & $84.6(22)[69.0-96.7]$ & 89.6 (43) [80.8-97.7] \\
\hline
\end{tabular}

Chi-square test for categorical data. ANOVA test for mean values. No significances between age groups and sex 


\section{Geriatric assessment}

Table 2 shows the results of the geriatric assessment.

The TTMC was completed successfully by $70.3 \%$ $(n=52)$ of the geriatric inpatients. The participants aged over 84 years passed the test more frequently than the younger ones. They needed between 21 and $347 \mathrm{~s}$ (mean, 90.7; $\mathrm{SD}=68.7 \mathrm{~s}$ ) to complete the test. The duration did not correlate by age $(r=0.07 ; p=0.553)$. Patients passing the TTMC needed significantly less time (67.2.; $\mathrm{SD}=42.6 \mathrm{~s})$ compared with those who failed (146.1; $\mathrm{SD}=86.0 \mathrm{~s} ; p=0.000)$. Three patients who passed the TTMC failed the NG. All age- or sex-related differences regarding the geriatric assessment results were without statistical significance.

Performance in activities of daily living and mobility assessed with the Barthel Index revealed that $77.0 \%(n=57)$ of the geriatric inpatients required medium- or high-level care. The need for high-level care was more prevalent in patients aged over 84 years and in males yet without statistical significance.

One third of the patients showed cognitive impairment assessed with the MMSE. Severe cognitive impairment was slightly more prevalent in patients aged over 84 years and in males.
Signs of depression were detected in one third of the geriatric patients by the GDS. Slight depression was more prevalent in males and moderate to major depression in females but without statistical significance.

\section{Plaque reduction}

Table 3 demonstrates the factors influencing plaque reduction on teeth and dentures in the participating geriatric inpatients. Performing tooth brushing and denture cleaning autonomously, patients reached reductions of mean TI (TI diff t0-t1) between 0.0 and 1.8 (mean 0.8; $\mathrm{SD}=0.5$ ) and of mean DHI (DHI diff $\mathrm{t} 0-\mathrm{t} 1$ ) between 0.0 and 0.8 (mean $0.2 ; \mathrm{SD}=0.2$ ). Female patients reached higher levels of plaque reduction on teeth compared with males.

The self-evaluation of oral hygiene did not coincide with the plaque reduction rates. Patients performing oral hygiene without help reached very slightly higher plaque reduction compared with those receiving help.

Plaque reduction was not significantly influenced by depression, assessed by GDS; level of care, evaluated by Barthel Scale; or cognitive impairment, measured by MMSE.

Passing the TTMC as well as the TTMC\&NG was significantly associated with better plaque removal on teeth and dentures (Table 3).

Table 2 Parameters of geriatric assessment tests in geriatric patients

\begin{tabular}{|c|c|c|c|c|c|}
\hline & All & Age $\leq 84$ years & Age $>84$ years & Males & Females \\
\hline \multicolumn{6}{|l|}{ TTMC } \\
\hline $\begin{array}{l}\text { TTMC passed \% }(n) \\
{[95 \% \mathrm{CI}]}\end{array}$ & $70.3(52)[59.5-81.0]$ & $61.5(24)[45.2-76.2]$ & $80.0(28)[65.4-91.7]$ & $69.2(18)[50.0-88.0]$ & $70.8(34)[57.1-83.3$ \\
\hline $\begin{array}{l}\text { TTMC\&NG passed } \\
\%(n)[95 \% \mathrm{CI}]\end{array}$ & $66.2(49)[55.4-75.7]$ & $61.5(24)[45.5-76.9]$ & $71.4(25)[65.3-86.2]$ & $69.2(18)[50.0-88.0]$ & $64.6(31)[50.0-78.3]$ \\
\hline \multicolumn{6}{|l|}{ Barthel Index } \\
\hline $\begin{array}{l}\text { Low-level care } \\
\%(n)[95 \% \text { CI] }\end{array}$ & $23.0(17)[13.5-33.8]$ & $25.6(10)[12.8-40.0]$ & $20.0(7)[8.3-35.3]$ & $19.2(5)[4.3-35.7]$ & $25.0(12)[13.2-37.0$ \\
\hline $\begin{array}{c}\text { Medium-level care } \\
\%(n)[95 \% \mathrm{CI}]\end{array}$ & $68.9(51)[58.1-79.7]$ & $69.2(27)[53.9-83.8]$ & $68.6(24)[52.4-82.5]$ & $69.2(18)[50.0-88.0]$ & $68.8(33)[56.1-81.6$ \\
\hline $\begin{array}{l}\text { High-level care } \\
\%(n)[95 \% \mathrm{CI}]\end{array}$ & $8.1(6)[2.7-14.9]$ & $5.1(2)[0.0-13.5]$ & $11.4(4)[2.6-23.3]$ & $11.5(3)[0.0-24.0]$ & $6.3(3)[0.0-14.0]$ \\
\hline \multicolumn{6}{|l|}{ MMSE } \\
\hline $\begin{array}{l}\text { No significant cognitive } \\
\text { impairment \% (n) }[95 \% \mathrm{CI}]\end{array}$ & $68.9(51)[58.1-79.7]$ & $64.1(25)[48.5-78.9]$ & $74.3(26)[60.0-88.6]$ & $69.2(18)[50.0-86.9]$ & $68.8(33)[54.3-81.8$ \\
\hline $\begin{array}{l}\text { Slight/moderate cognitive } \\
\text { impairment \% (n) }[95 \% \mathrm{CI}]\end{array}$ & $18.9(14)[10.8-29.7]$ & $25.6(10)[12.5-41.2]$ & $11.4(4)[2.8-23.1]$ & $15.4(4)[30.5-30.4]$ & $20.8(10)[10.0-34.6$ \\
\hline $\begin{array}{l}\text { Severe cognitive impairment } \\
\%(n)[95 \% \mathrm{CI}]\end{array}$ & $12.2(9)[5.4-20.2]$ & 10.3 (4) $[2.3-20.5]$ & $14.3(5)[3.1-25.8]$ & 15.4 (4) $[3.0-30.8]$ & $10.4(5)[2.2-19.5]$ \\
\hline \multicolumn{6}{|l|}{ Geriatric Depression Scale (GDS) } \\
\hline No depression $\%(n)[95 \% \mathrm{CI}]$ & $64.9(48)[54.1-75.7]$ & $64.1(25)[47.5-79.1]$ & $65.7(23)[48.8-81.6]$ & $57.7(15)[37.9-75.8]$ & $68.8(33)[55.6-81.3$ \\
\hline Slight depression \% (n) $[95 \% \mathrm{CI}]$ & $27.0(20)[17.6-36.5]$ & $25.6(10)[11.8-40.9]$ & $28.6(10)[14.3-44.1]$ & $38.5(10)[21.7-57.1]$ & $20.8(10)[8.7-32.0]$ \\
\hline $\begin{array}{l}\text { Moderate/major depression } \\
\%(n)[95 \% \mathrm{CI}]\end{array}$ & 8.1 (6) [2.7-14.9] & $10.3(4)[2.4-21.2]$ & $5.7(2)[0.0-15.1]$ & $3.8(1)[0.0-12.5]$ & $10.4(5)[2.4-20.4]$ \\
\hline
\end{tabular}

*Chi-square test, no significances between age groups and sex 
Table 3 Factors influencing plaque reduction on teeth (measured by TI) and on dentures (measured by DHI) in geriatric inpatients

\begin{tabular}{|c|c|c|c|c|c|}
\hline Factor & & $\begin{array}{l}\text { TI diff } \mathrm{t} 0-\mathrm{t} 1 \\
\text { mean }(\mathrm{SD})\end{array}$ & $p^{*}$ & $\begin{array}{l}\text { DHI diff } \mathrm{t} 0-\mathrm{t} 1 \\
\text { mean (SD) }\end{array}$ & $p^{*}$ \\
\hline Study population & & $0.8(0.5)$ & & $0.2(0.2)$ & \\
\hline Age & $\begin{array}{l}<84 \text { years } \\
\geq 85 \text { years }\end{array}$ & $\begin{array}{l}0.8(0.2) \\
0.9(0.2)\end{array}$ & 0.319 & $\begin{array}{l}0.2(0.2) \\
0.3(0.2)\end{array}$ & 0.115 \\
\hline Sex & $\begin{array}{l}\text { Males } \\
\text { Females }\end{array}$ & $\begin{array}{l}0.9(0.4) \\
0.3(0.2)\end{array}$ & 0.393 & $\begin{array}{l}0.2(0.2) \\
0.3(0.2)\end{array}$ & 0.191 \\
\hline Oral hygiene (self-evaluation) & $\begin{array}{l}\text { Very good } \\
\text { Good }\end{array}$ & $\begin{array}{l}0.3(0.3) \\
0.9(0.4)\end{array}$ & 0.129 & $\begin{array}{l}0.3(0.3) \\
0.3(0.2)\end{array}$ & 0.485 \\
\hline & Satisfactory & $0.8(0.5)$ & & $0.2(0.2)$ & \\
\hline Subjective problems in performing oral hygiene & $\begin{array}{l}\text { Yes } \\
\text { No }\end{array}$ & $\begin{array}{l}1.1(0.4) \\
0.8(0.5)\end{array}$ & 0.111 & $\begin{array}{l}0.3(0.2) \\
0.2(0.2)\end{array}$ & 0.192 \\
\hline Performing oral hygiene without help & $\begin{array}{l}\text { Yes } \\
\text { No }\end{array}$ & $\begin{array}{l}0.8(0.5) \\
0.9(0.4)\end{array}$ & 0.682 & $\begin{array}{l}0.2(0.2) \\
0.3(0.2)\end{array}$ & 0.432 \\
\hline TTMC time cutoffs & $\begin{array}{l}<45 \mathrm{~s} \\
45-70 \mathrm{~s} \\
>70 \mathrm{~s}\end{array}$ & $\begin{array}{l}0.9(0.6) \\
1.0(0.4) \\
0.5(0.3)\end{array}$ & 0.003 & $\begin{array}{l}0.3(0.2) \\
0.3(0.2) \\
0.2(0.1)\end{array}$ & 0.081 \\
\hline TTMC & $\begin{array}{l}\text { Passed } \\
\text { Failed }\end{array}$ & $\begin{array}{l}0.9(0.5) \\
0.6(0.3)\end{array}$ & 0.016 & $\begin{array}{l}0.3(0.2) \\
0.2(0.1)\end{array}$ & 0.031 \\
\hline TTMC\&NG & $\begin{array}{l}\text { Passed } \\
\text { Failed }\end{array}$ & $\begin{array}{l}0.9(0.4) \\
0.5(0.3)\end{array}$ & 0.015 & $\begin{array}{l}0.3(0.2) \\
0.2(0.1)\end{array}$ & 0.014 \\
\hline Barthel Scale & $\begin{array}{l}\text { Low-level care } \\
\text { Medium-level care } \\
\text { High-level care }\end{array}$ & $\begin{array}{l}0.8(0.5) \\
0.8(0.4) \\
0.7(0.5)\end{array}$ & 0.887 & $\begin{array}{l}0.3(0.2) \\
0.2(0.2) \\
0.2(0.1)\end{array}$ & 0.412 \\
\hline MMSE & $\begin{array}{l}\text { No significant cognitive impairment } \\
\text { Slight/moderate cognitive impairment } \\
\text { Severe cognitive impairment }\end{array}$ & $\begin{array}{l}0.8(0.4) \\
1.0(0.6) \\
0.7(0.3)\end{array}$ & 0.300 & $\begin{array}{l}0.3(0.2) \\
0.3(0.2) \\
0.2(0.2)\end{array}$ & 0.550 \\
\hline Geriatric Depression Scale (GDS) & $\begin{array}{l}\text { No depression } \\
\text { Slight depression } \\
\text { Moderate/major depression }\end{array}$ & $\begin{array}{l}0.8(0.5) \\
0.8(0.3) \\
1.0(0.7)\end{array}$ & 0.653 & $\begin{array}{l}0.3(0.2) \\
0.2(0.1) \\
0.4(0.2)\end{array}$ & 0.089 \\
\hline
\end{tabular}

*ANOVA test. Significant $p$-values are displayed in bold

There was observed a weak correlation between the time needed to complete the test and the plaque reduction on teeth $(r=-0.3, p=0.028$, Spearman's correlation test) and dentures $(r=-0.2, p=0.090$, Spearman's correlation test). However, using the cutoffs of the TTMC, patients needing more than $70 \mathrm{~s}$ to complete the test had lower plaque reduction rates on teeth $(p=0.003$; ANOVA $)$ and dentures $(p=0.081$; ANOVA) (Table 3).

The occurrence of problems during the performance of TTMC was significantly associated with less plaque removal only for cognitive and visual problems (Table 4). In patients with cognitive problems during TTMC, significantly lower plaque reduction was observed for tooth brushing and denture cleaning. Additionally, visual problems were associated with significantly lower plaque removal on dentures. All other problems did not lead to significant lower plaque reduction rates (Table 4$)$.

\section{Validation}

The mean difference of the plaque index between $\mathrm{t} 0$ and $\mathrm{t} 1$ was used to group the plaque reduction rates in above and below average. Passing the TTMC\&NG was significantly associated with above average plaque reduction on teeth ( $p=0.044$, chi-square test) and dentures $(p=0.033$, chisquare test). $2 \times 2$ tables (Table 5 ) were used to calculate SE, SP, NPV, and PPV of the TTMC \&NG and its time cutoffs for above and below average plaque reduction. The TTMC\&NG had a higher sensitivity for above average plaque removal on teeth $(86.4 \%)$ than on dentures (77.8\%). Failing the test predicts with high probability below average tooth and denture plaque reduction (NPV $75.0 \%$ and $72.7 \%$, respectively). The time cutoff of the TTMC failed to produce higher test results compared with the TTMC\&NG, except the NPV for plaque reduction on dentures $(84.4 \%$ vs. $72.7 \%$, respectively). 
Table 4 Problems during TTMC\&NG influencing plaque reduction on teeth (measured by TI) and on dentures (measured by DHI) in geriatric patients

\begin{tabular}{llllllll}
\hline $\begin{array}{l}\text { Problems occurring during } \\
\text { TTMC\&NG }\end{array}$ & & $n$ & $\begin{array}{l}\text { TI diff t0-t1 } \\
\text { mean (SD) }\end{array}$ & $p^{*}$ & $n$ & $\begin{array}{l}\text { DHI diff t0-t1 } \\
\text { mean (SD) }\end{array}$ & $p^{*}$ \\
\hline Any problems during TTMC & Yes & 32 & $0.9(0.3)$ & 0.280 & 44 & $0.2(0.2)$ & 0.435 \\
& No & 12 & $0.8(0.5)$ & & 16 & $0.3(0.2)$ & \\
Fine motor skill problems & Yes & 22 & $0.8(0.5)$ & 0.519 & 29 & $0.3(0.2)$ & 0.774 \\
& No & 22 & $0.9(0.4)$ & & 31 & $0.2(0.2)$ & \\
Gross motor skill problems & Yes & 16 & $0.8(0.5)$ & 0.621 & 21 & $0.3(0.2)$ & 0.183 \\
& No & 28 & $0.8(0.4)$ & & 39 & $0.2(0.2)$ & \\
Cognitive problems & Yes & 12 & $0.5(0.3)$ & $\mathbf{0 . 0 0 7}$ & 22 & $0.2(0.1)$ & $\mathbf{0 . 0 0 9}$ \\
& No & 32 & $0.9(0.4)$ & & 38 & $0.3(0.2)$ & \\
Visual problems & Yes & 14 & $0.7(0.5)$ & 0.167 & 22 & $0.2(0.1)$ & $\mathbf{0 . 0 1 7}$ \\
\multirow{2}{*}{ Painful shoulder motion } & No & 30 & $0.9(0.4)$ & & 38 & $0.3(0.2)$ & \\
& Yes & 17 & $0.8(0.5)$ & 0.946 & 24 & $0.2(0.2)$ & 0.719 \\
& No & 27 & $0.8(0.5)$ & & 36 & $0.3(0.2)$ & \\
\hline
\end{tabular}

*ANOVA test. Significant values are displayed in bold

\section{Discussion}

To the author's knowledge, this is the first study evaluating the predictability of effective tooth brushing and denture cleaning by using an established geriatric assessment toolthe TTMC - complemented with testing the range of shoulder mobility by simply griping the backside of the neck.

The TTMC\&NG seems to be a suitable tool to predict the ability of geriatric inpatients to perform autonomously effective oral hygiene. The high sensitivity shows that the large majority of participants reaching above average plaque reduction also passed the TTMC\&NG. Furthermore, the high NPV demonstrated that participants failing the test reached only below average plaque reductions with high probability. Therefore, the TTMC\&NG had a better potential to detect geriatric inpatients with deficiencies in performing effective oral hygiene independently compared with the $70 \mathrm{~s}$ time cutoff of the TTMC.

Substantial advantages of the TTMC\&NG were the simple and short procedure $[18,19]$. The expenditure of time is only about $5 \mathrm{~min}$, and the test might be performed within the geriatric assessment, as all geriatric inpatients pass comprehensive geriatric assessment at admission to the geriatric department. Good reliability and validity of the TTMC was already reported [18]. The TTMC requires practical skills relevant to activities of daily living and specifically to oral hygiene: gross and fine motor skills of hand and fingers, visual skills, and cognitive and executive function [19]. Additionally, sufficient mobility of the shoulder is necessary to raise a toothbrush into the mouth and to execute brushing movements. Therefore, the TTMC was complemented with a test for range of shoulder mobility. This test included as a short and simple exercise the griping of the own backside of the neck. The expenditure of time is less than $1 \mathrm{~min}$, and impairment of shoulder motility can be easily detected.

Dentists reported difficulties in estimating the ability on cognitively impaired older patients to perform oral hygiene and desired a reliable and easy-to-use assessment tool [17]. The "Dental Activities Test (DAT)" [32] was proposed as a reliable and valid clinical tool for measuring dentally related function in cognitively impaired older adults. The DAT was moderately associated with dental plaque on teeth and not with plaque on dentures [32]. The correlation between the DAT scores and the ability of plaque reduction is not reported.

Table $52 \times 2$ tables contrasting the passing/failure of the TTMC\&NG with above and below average plaque reduction rates in geriatric patients

\begin{tabular}{lllll}
\hline & $\begin{array}{l}\text { TTMC\&NG passed } n(\%) \\
\text { [positive outcome] }\end{array}$ & $\begin{array}{l}\text { TTMC\&NG failed } n(\%) \\
\text { [negative outcome] }\end{array}$ & $\begin{array}{l}<70 \mathrm{~s} n(\%) \\
\text { [positive outcome] }\end{array}$ & $\begin{array}{l}>70 \mathrm{~s} \mathrm{n}(\%) \\
\text { [negative outcome] }\end{array}$ \\
\hline $\begin{array}{c}\text { Plaque reduction on teeth } \\
\text { Above average* [positive outcome] }\end{array}$ & $19(43.2)$ & $3(6.8)$ & $19(43.2)$ & $3(6.8)$ \\
Below average* [negative outcome] & $13(29.5)$ & $9(20.5)$ & $11(25.0)$ & $11(25.0)$ \\
$\begin{array}{c}\text { Plaque reduction on dentures } \\
\text { Above average\# [positive outcome] }\end{array}$ & $21(33.3)$ & $6(9.5)$ & $14(23.3)$ & $18(30.0)$ \\
Below average\# [negative outcome] & $20(31.7)$ & $16(25.4)$ & $18(15.0)$ \\
\hline
\end{tabular}

* Mean TI diff $\mathrm{t} 0-\mathrm{t} 1=0.84$; \#mean DHI diff $\mathrm{t} 0-\mathrm{t} 1=0.24$ 
Furthermore, this tool was designed for use in dental environments and requires dentally trained staff for conducting and interpreting the test. In contrast to the DAT, the proposed TTMC\&NG does not require dental environment or staff and is therefore suitable to be performed in standard medical settings like geriatric clinics, hospitals, nursing homes, or medical doctor's surgeries.

The self-perceived quality of oral hygiene, reported by geriatric inpatients, did not correlate with the actual ability to perform plaque reduction. The standard geriatric assessment tests such as MMSE, GDS, and Barthel Score were not able to detect geriatric inpatients with deficiencies in performing oral hygiene. The Resident Assessment Instrument (RAI), a comprehensive, valid, and reliable tool to assess geriatric residents' clinical and functional status to monitor and improve quality of care, might reveal information to evaluate geriatric resident's competence to perform autonomously effective tooth and denture cleaning. This shall be subject of further study although the RAI assesses oral health-related issues with low reliability [33] and validity $[34,35]$ underestimating oral problems and lacking to record dental plaque.

The amount of time needed to perform the TTMC played a minor role to detect deficient oral hygiene in geriatric inpatients. More important than the time needed to perform the TTMC were the consideration of problems occurring during test performance. Except the painful shoulder motion, all observed problems were associated with higher frequency to fail the TTMC\&NG. It seems that patients did not decline oral hygiene due to pain experience in the shoulder. Missing or limited shoulder mobility instead hindered tooth brushing. Furthermore, cognitive and visual impairment were associated with lower plaque reduction rates. Inconsistently, patients with lower MMSE did not show lower plaque reduction rates. It is assumed that the MMSE has low sensitivity to detect patients with cognitive impairment affecting the performance of oral hygiene. This observation is in line with a recent systematic review reporting about the inconsistent findings regarding the interaction between cognitive impairment and oral health $[36,37]$.

Estimating the ability of performing oral hygiene with the TTMC\&NG should consider individual coping skills of the patients. Geriatric inpatients might have developed routines or behaviors compensating impaired functions. For example, in this study, two female patients with very low visual skills performed the TTMC quickly and confidently, because both were working their whole life as sales clerk and counting money was a more familiar exercise than tooth brushing. In these cases, the predictability of the TTMC regarding the ability for oral hygiene is less reliable.

Compared with representative older German people aged between 75 and 100 years in need of care [8], geriatric inpatients had slightly higher caries experience (26.3 vs. 24.5$)$, lower prevalence of edentulism (37.8\% vs. $53.7 \%$ ), and lower number of natural teeth (6.9 vs. 5.6) [8]. Normal ability to perform oral hygiene was observed in $22.5 \%$ of $75-100$ year-old persons in need for care [8], which is in contrast to our finding of $70.2 \%$ patients passing the TTMC\&NG. In that representative study, the ability to perform oral hygiene was evaluated by dentists involved in the oral examination of the patients. The evaluation was based solely on the dentist's professional perception, without use of any competence test. If dentists acknowledged difficulties in estimating the ability of impaired older patients to perform oral hygiene [17], it can be assumed that non-dental professionals like geriatric medical staff encounter comparable or even more difficulties to distinguish between patients with and without need for help and support for oral hygiene by nursing staff or nursing relatives. Although the TTMC\&NG is not generally used in every geriatric hospital, it has the potential to support dental and nondental professionals in this decision process, since performance and interpretation of the test does not require dental knowledge.

Nursing staff or nursing relatives play a crucial role to safeguard oral hygiene and oral health in geriatric patients. Therefore, it is mandatory to raise attention, responsibility, knowledge, and motivation towards oral problems in caregivers and to empower them to face this task.

Several limitations of this study are to be considered. First, the study was conducted in the limited setting of one geriatric clinic. Therefore, generalization and transferability to other settings should be analyzed in further investigations. Second, due to limited personal resources, the same dentist conducted the TTMC\&NG and the oral examinations, which could be considered a risk for observation bias. In further studies, it would be preferable to have two blinded investigators, one running the TTMC\&NG and another conducting the oral examinations. Third, the TTMC\&NG was performed once, failing to detect performance variations due to changes in the general health status and environment. When predicting the ability to perform oral hygiene, it should be considered that passing the test does not necessarily imply good quality oral hygiene, especially in patients with acute medical conditions, inadequate caregiver support, or low oral health literacy. Although direct observation of performing the TTMC\&NG may clarify discrepancies between observations and selfand proxy perceptions of the patients' abilities, the TTMC\&NG did not detect the everyday performance in the home environment.

\section{Conclusion}

The TTMC\&NG served as a suitable predictor for the ability of geriatric inpatients to perform autonomously effective tooth brushing and denture cleaning. It might help geriatric medical 
staff to identify geriatric inpatients unable to perform effective oral hygiene by themselves.

Acknowledgments The authors thank the medical staff and nurses from the Geriatric Clinic of Jena University Hospital for assistance to conduct this study and the geriatric inpatients for participation.

Funding information Open Access funding provided by Projekt DEAL. The work was supported by the Section of Preventive and Pediatric Dentistry, Department of Orthodontics, Jena University Hospital, Germany.

\section{Compliance with ethical standards}

Conflict of interest Ina Manuela Schüler declares that she has no conflict of interest. Barbara Kurtz declares that she has no conflict of interest. Roswitha Heinrich-Weltzien declares that she has no conflict of interest. Thomas Lehmann declares that he has no conflict of interest. Anja Kwetkat declares that she has no conflict of interest.

Ethical approval All procedures performed in this study involving human participants were in accordance with the ethical standards of the institutional research committee and with the 1964 Helsinki declaration and its later amendments or comparable ethical standards. Ethical approval was received by the Ethics Committee of Jena University Hospital (4590-11/15). The study was registered at the German Registry of Clinical Trials (DRKS00011095).

Informed consent All patients or their legal guardians gave written informed consent to participate in the study and to provide access to medical records.

Open Access This article is licensed under a Creative Commons Attribution 4.0 International License, which permits use, sharing, adaptation, distribution and reproduction in any medium or format, as long as you give appropriate credit to the original author(s) and the source, provide a link to the Creative Commons licence, and indicate if changes were made. The images or other third party material in this article are included in the article's Creative Commons licence, unless indicated otherwise in a credit line to the material. If material is not included in the article's Creative Commons licence and your intended use is not permitted by statutory regulation or exceeds the permitted use, you will need to obtain permission directly from the copyright holder. To view a copy of this licence, visit http://creativecommons.org/licenses/by/4.0/.

\section{References}

1. Humphrey LL, Fu R, Buckley DI, Freeman M, Helfand M (2008) Periodontal disease and coronary heart disease incidence: a systematic review and meta-analysis. J Gen Intern Med 23(12):2079-2086

2. Azarpazhooh A, Leake JL (2006) Systematic review of the association between respiratory diseases and oral health. J Periodontol 77(9):1465-1482

3. Moynihan PJ (2007) The relationship between nutrition and systemic and oral well-being in older people. J Am Dent Assoc 138(4): 493-497

4. Lee KH, Wu B, Plassman BL (2013) Cognitive function and oral health-related quality of life in older adults. J Am Geriatr Soc 61(9): $1602-1607$
5. Sanderink RB, Schlagenhauf U (2013) Beschleunigen parodontale Entzündungen den Alterungsprozess des menschlichen Körpers Das Inflammaging Konzept. Dtsch Zahnnärztl Z 68:416-425

6. Murray Thomson W (2014) Epidemiology of oral health conditions in older people. Gerodontology 31(Suppl 1):9-16

7. Petersen PE, Kandelman D, Arpin S, Ogawa H (2010) Global oral health of older people-call for public health action. Community Dent Health 27(4 Suppl 2):257-267

8. Jordan R, W M (2016) Fünfte Deutsche Mundgesundheitsstudie (DMS V). Institut der Deutschen Zahnärzte, Deutscher Zahnärzteverlag, Köln

9. van der Putten GJ, de Baat C, de Visschere L, Schols J (2014) Poor oral health, a potential new geriatric syndrome. Gerodontology 31(Suppl 1):17-24

10. Shay K (2002) Infectious complications of dental and periodontal diseases in the elderly population. Clin Infect Dis 34(9):1215-1223

11. Peltola P, Vehkalahti MM, Simoila R (2005) Oral health-related well-being of the long-term hospitalised elderly. Gerodontology 22(1):17-23

12. Scannapieco FA (1999) Role of oral bacteria in respiratory infection. J Periodontol 70(7):793-802

13. Bansal M, Khatri M, Taneja V (2013) Potential role of periodontal infection in respiratory diseases - a review. J Med Life 6(3):244 248

14. Sjogren P et al (2008) A systematic review of the preventive effect of oral hygiene on pneumonia and respiratory tract infection in elderly people in hospitals and nursing homes: effect estimates and methodological quality of randomized controlled trials. J Am Geriatr Soc 56(11):2124-2130

15. Muller F (2015) Oral hygiene reduces the mortality from aspiration pneumonia in frail elders. J Dent Res 94(3 Suppl):14S-16S

16. Scannapieco FA, Shay K (2014) Oral health disparities in older adults: oral bacteria, inflammation, and aspiration pneumonia. Dent Clin N Am 58(4):771-782

17. Chen X, Clark JJJ (2013) Assessment of dentally-related functional competency for older adults with cognitive impairment - a survey for special-care dental professionals. Special Care Dentist 33(2): $48-55$

18. Nikolaus T, Bach M, Oster P, Schlierf G (1995) The Timed Test of Money Counting: a simple method of recognizing geriatric patients at risk for increased health care. Aging (Milano) 7(3):179-183

19. Zeyfang A et al (2012) A short easy test can detect ability for autonomous insulin injection by the elderly with diabetes mellitus. J Am Med Dir Assoc 13(1):81.e15-81.e18

20. WHO, Oral health surveys: basic methods (4th ed.). Geneva: World Health Organisation, 1997

21. Meyle J, Jepsen S (2000) Der parodontale Screening Index (PSI). Parodontologie 11(1):17-21

22. Turesky S, Gilmore ND, Glickman I (1970) Reduced plaque formation by the chloromethyl analogue of victamine C. J Periodontol 41(1):41-43

23. Wefers K (1999) Der "Denture Hygiene Index" DHI. Dental Forum $1: 13-15$

24. MDK (2017) Assessment in der Geriatrie. Kompetenzzentrum Geriatrie

25. Stuck AE, Iliffe S (2011) Comprehensive geriatric assessment for older adults. BMJ 343:d6799

26. Wade DT, Collin C (1988) The Barthel ADL Index: a standard measure of physical disability? Int Disabil Stud 10(2):64-67

27. Yesavage JA, Brink TL, Rose TL, Lum O, Huang V, Adey M, Leirer VO (1982) Development and validation of a geriatric depression screening scale: a preliminary report. J Psychiatr Res 17(1):3749

28. Folstein MF, Folstein SE, McHugh PR (1975) "Mini-mental state". A practical method for grading the cognitive state of patients for the clinician. J Psychiatr Res 12(3):189-198 
29. WHO (2018) International Statistical Classification of Diseases and Related Health Problems ICD-10, U50-U52

30. Tombaugh TN, McIntyre NJ (1992) The mini-mental state examination: a comprehensive review. J Am Geriatr Soc 40(9):922-935

31. Almeida OP, Almeida SA (1999) Short versions of the geriatric depression scale: a study of their validity for the diagnosis of a major depressive episode according to ICD-10 and DSM-IV. Int J Geriatr Psychiatry 14(10):858-865

32. Chen X, Zimmerman S, Potter GG, Sloane PD, Cohen LW, Reed D (2017) Assessment of dentally related function in individuals with cognitive impairment: the dental activities test. J Am Geriatr Soc 65(3):580-585

33. Hawes C, Morris JN, Phillips CD, Mor V, Fries BE, Nonemaker S (1995) Reliability estimates for the minimum data set for nursing home resident assessment and care screening (MDS). Gerontologist 35(2):172-178
34. Hoben $\mathrm{M}$ et al (2016) Oral/dental items in the resident assessment instrument - minimum Data Set 2.0 lack validity: results of a retrospective, longitudinal validation study. Population Health Metrics 14(1):36

35. Folse GJ (2001) National MDS and dental deficiency data reported by the US Health Care Financing Administration (HCFA). Spec Care Dentist 21(1):37-38

36. Wu B, Fillenbaum GG, Plassman BL, Guo L (2016) Association between oral health and cognitive status: a systematic review. J Am Geriatr Soc 64(4):739-751

37. Chen X, Clark JJ, Chen H, Naorungroj S (2015) Cognitive impairment, oral self-care function and dental caries severity in community-dwelling older adults. Gerodontology 32(1):53-61

Publisher's note Springer Nature remains neutral with regard to jurisdictional claims in published maps and institutional affiliations. 